\title{
Nonclassicality tests by classical bounds on the statistics of multiple outcomes
}

\author{
Alfredo Luis* \\ Departamento de Óptica, Facultad de Ciencias Físicas, Universidad Complutense, 28040 Madrid, Spain
}

(Received 20 June 2010; published 18 August 2010)

\begin{abstract}
We derive simple practical tests revealing the quantum nature of states by the violation of classical upper bounds on the statistics of multiple outcomes of an observable. These criteria can be expressed in terms of the Kullback-Leibler divergence (or relative entropy). Nonclassicality tests for multiple outcomes can be satisfied by states that do not fulfill the corresponding single-outcome criteria.
\end{abstract}

Introduction. Nonclassicality is a key concept supporting the necessity of the quantum theory [1]. In a previous work we derived simple and robust practical tests to reveal the quantum nature of states by breaking the classical bound on the probability of a single outcome of an observable [2]. In this work we generalize this to the probability of multiple outcomes of the same measurement.

We show that these tests can be expressed in terms of the Kullback-Leibler divergence, comparing the actual observed statistics with the closest statistics compatible with classical physics. Moreover, we show that multiple-outcome nonclassicality tests are more powerful than the single-outcome ones, since they can be satisfied by states that do not fulfill the corresponding single-outcome criteria. Thus, this approach extends the range of nonclassical states that can be disclosed by these simple procedures.

The multiple-outcome approach preserves the same good properties of the single-outcome case [2]: it can be applied to arbitrary measurements (in sharp contrast with other criteria that refer exclusively to specific schemes), data analysis is reduced to a minimum (definite conclusions can be obtained without evaluation of moments or any other data analysis), and it has a robustness under practical imperfections.

We derive the classical bounds to the probability of multiple outcomes of the same measurement, expressing them in terms of the Kullback-Leibler divergence. We also show that the multiple-outcome approach is consistent with the singleoutcome situation, which is retrieved as a particular case. Finally, the main distinctive features of the multiple-outcome approach are discussed.

Classical bounds for multiple outcomes. We consider the statistics of $M$ outcomes of a given measured observable. Since we are going to compare probabilities, we assume that the number $N$ of repetitions of the measurement is high enough so that

$$
p(m \mid \rho) \rightarrow \frac{n_{m}}{N}
$$

where $p(m \mid \rho), m=1, \ldots, M$, is the probability of the outcome $m$ when the state is $\rho$, and $n_{m}$ is the number of times of occurrence of the outcome $m$, with

$$
\sum_{m=1}^{M} n_{m}=N
$$

\footnotetext{
*alluis@ fis.ucm.es; URL: http://www.ucm.es/info/gioq
}

The statistics $n_{m}$ can be represented by the $M$-dimensional vector $\boldsymbol{n}=\left(n_{1}, \ldots, n_{M}\right)$. We call $P(\boldsymbol{n} \mid \rho)$ the probability of $\boldsymbol{n}$ when the state is $\rho$

$$
P(\boldsymbol{n} \mid \rho)=N ! \Pi_{m=1}^{M} \frac{[p(m \mid \rho)]^{n_{m}}}{n_{m} !} .
$$

The nonclassical tests addressed in this work are derived from the following simple reasoning: all classical states $\Phi$ satisfy the inequality

$$
P(\boldsymbol{n} \mid \Phi) \leqslant P\left(\boldsymbol{n} \mid \Phi_{n}\right),
$$

where $\Phi_{n}$ is the classical state that maximizes $P(\boldsymbol{n} \mid \Phi)$ when $\Phi$ runs all classical states. Therefore, we get the following nonclassical criteria:

$$
P(\boldsymbol{n} \mid \rho)>P\left(\boldsymbol{n} \mid \Phi_{n}\right) \rightarrow \rho \text { is nonclassical. }
$$

The application of these tests crucially depends on the definition of what are classical states. The key point is that there is no unique definition. In this work we adopt the most widely used approach, where a state is classical if its Glauber-Sudarshan phase-space $P$ representation [3] exhibits all the properties of a classical probability density. For nonclassical states, $P$ takes negative values or fails to be a proper function, becoming more singular than a delta function. Some other correspondences between states and phase-space distributions may be adopted [4], leading in general to different conclusions [5].

Kullback-Leibler divergence. Let us express the criteria (5) in a different form. Taking into account explicitly that $n_{m}=$ $N p(m \mid \rho)$, we have

$$
P\left(\boldsymbol{n} \mid \Phi_{n}\right)=N ! \Pi_{m=1}^{M} \frac{\left[p\left(m \mid \Phi_{n}\right)\right]^{N p(m \mid \rho)}}{\Gamma[N p(m \mid \rho)+1]},
$$

and similarly

$$
P(\boldsymbol{n} \mid \rho)=N ! \Pi_{m=1}^{M} \frac{[p(m \mid \rho)]^{N p(m \mid \rho)}}{\Gamma[N p(m \mid \rho)+1]} .
$$

Their quotient is

$$
\frac{P\left(\boldsymbol{n} \mid \Phi_{n}\right)}{P(\boldsymbol{n} \mid \rho)}=\exp \left[-N K\left(\rho \| \Phi_{n}\right)\right],
$$

where $K\left(\rho \| \Phi_{n}\right)$ is the Kullback-Leibler divergence (or relative entropy) [6]

$$
K\left(\rho \| \Phi_{n}\right)=\sum_{m=1}^{M} p(m \mid \rho) \ln \frac{p(m \mid \rho)}{p\left(m \mid \Phi_{n}\right)} .
$$


This is the relative probability of obtaining the statistics $\boldsymbol{n}$ corresponding to the state $\rho$ if the system is in the optimum classical state $\Phi_{n}$. In other words, $K$ measures the compatibility of the actual observed statistics $\boldsymbol{n}$ with the optimum classical hypothesis $\Phi_{n}$ that minimizes $K(\rho \| \Phi)$. After Eq. (8) we can reformulate criteria (5) as

$$
K\left(\rho \| \Phi_{n}\right)>0 \rightarrow \rho \text { is nonclassical. }
$$

Since $K\left(\rho \| \Phi_{n}\right)$ measures how far the actual statistics are from the classical domain (depending on the observable considered), it may be adopted as a kind of degree of nonclassicality. This would be related to a previously introduced nonclassicality measure based on relative entropy between states, which does not depend on any definite observable [7]. Some other measures of nonclassicality can be found in Ref. [8].

Compatibility with single-outcome criteria. The minimum number of outcomes is $M=2, \boldsymbol{n}=(n, N-n)$, with $n=$ $N p(1 \mid \rho), p(2 \mid \rho)=1-p(1 \mid \rho)$, and

$$
P(\boldsymbol{n} \mid \rho)=\left(\begin{array}{l}
N \\
n
\end{array}\right)[p(1 \mid \rho)]^{n}[1-p(1 \mid \rho)]^{N-n} .
$$

Let us demonstrate that the criteria (5) is equivalent to the single-outcome criteria in Ref. [2], i.e.,

$$
p(1 \mid \rho)>p\left(1 \mid \Phi_{1}\right) \rightarrow \rho \text { nonclassical, }
$$

where $\Phi_{1}$ is the classical state that maximizes $p(1 \mid \Phi)$.

First, let us prove that

$$
p(1 \mid \rho)>p\left(1 \mid \Phi_{1}\right) \rightarrow P(\boldsymbol{n} \mid \rho)>P\left(\boldsymbol{n} \mid \Phi_{n}\right) .
$$

This holds since the actual probability $p(1 \mid \rho)=n / N$ gives the maximum of $P(\boldsymbol{n} \mid \rho)$ in Eq. (11) when $p(1 \mid \rho)$ is varied. Thus, if $p(1 \mid \rho)>p(1 \mid \Phi)$ for all classical $\Phi$ then $p(1 \mid \rho) \neq$ $p(1 \mid \Phi)$ and $P(\boldsymbol{n} \mid \rho)>P(\boldsymbol{n} \mid \Phi)$ for all $\Phi$, so that Eq. (13) follows.

Let us address the converse implication

$$
P(\boldsymbol{n} \mid \rho)>P\left(\boldsymbol{n} \mid \Phi_{n}\right) \rightarrow p(1 \mid \rho)>p\left(1 \mid \Phi_{1}\right) .
$$

If $P(\boldsymbol{n} \mid \rho)>P\left(\boldsymbol{n} \mid \Phi_{n}\right)$ then $p(1 \mid \Phi) \neq p(1 \mid \rho)$ for all classical states $\Phi$, so that either $p(1 \mid \rho)>p(1 \mid \Phi)$ or $p(1 \mid \rho)<$ $p(1 \mid \Phi)$ which means $p(2 \mid \rho)>p(2 \mid \Phi)$. In order to show that $p(1 \mid \rho)>p(1 \mid \Phi)$ or $p(2 \mid \rho)>p(2 \mid \Phi)$ holds simultaneously for all classical $\Phi$ we can use that the set of probabilities $p(1 \mid \Phi)$ is connected, because for $\Phi_{\lambda}=\lambda \Phi_{\alpha}+(1-\lambda) \Phi_{\beta}$ we get $p\left(1 \mid \Phi_{\lambda}\right)=\lambda p\left(1 \mid \Phi_{\alpha}\right)+(1-\lambda) p\left(1 \mid \Phi_{\beta}\right)$. Therefore for $p(1 \mid \Phi) \neq p(1 \mid \rho)$ connection implies either $p(1 \mid \rho)>p(1 \mid \Phi)$ or $p(1 \mid \rho)<p(1 \mid \Phi)$ for all $\Phi$, so that Eq. (14) holds directly if $p(1 \mid \rho)>p(1 \mid \Phi)$ or after a suitable label exchange $1 \leftrightarrow 2$ if $p(1 \mid \rho)<p(1 \mid \Phi)$.

Distinctive features. Besides being a generalization, the multiple-outcome approach presents distinctive features compared to the single-outcome scheme in Ref. [2]. This is because the global maximization of $P(\boldsymbol{n} \mid \Phi)$ is different from the independent maximization of the individual $p(m \mid \Phi)$, which leads in general to a different optimum classical $\Phi_{m}$ for each outcome. Next we show two relevant differences.
1. Optimum classical states can be mixed: The classical state maximizing the single-outcome probability $p(m \mid \Phi)$ is a pure coherent state $\Phi_{m}=\left|\alpha_{m}\right\rangle\left\langle\alpha_{m}\right|$, since for all classical states

$$
\Phi=\int d^{2} \alpha P(\alpha)|\alpha\rangle\langle\alpha|, \quad P(\alpha) \geqslant 0,
$$

we have

$\left.\left.p(m \mid \Phi)=\int d^{2} \alpha P(\alpha) p(m|| \alpha\rangle\langle\alpha|\right) \leqslant p\left(m|| \alpha_{m}\right\rangle\left\langle\alpha_{m}\right|\right)$,

where $\alpha_{m}$ is the complex amplitude of the coherent state maximizing $p(m|| \alpha\rangle\langle\alpha|)$. The equality in Eq. (16) is reached for the pure coherent state $\Phi=\left|\alpha_{m}\right\rangle\left\langle\alpha_{m}\right|$ so that $P(\alpha)=$ $\delta^{(2)}\left(\alpha-\alpha_{m}\right)$. However, this need not be the case when maximizing $P(\boldsymbol{n} \mid \Phi)$, and the maximum can be obtained for mixed classical states.

As a simple example revealing this feature, let us consider the measurement of the number of photons of a single-mode radiation field in the squeezed vacuum state [1]

$p(2 m \mid \rho)=\frac{1}{\cosh r} \frac{(2 m) !}{m !^{2}}\left(\frac{1}{2} \tanh r\right)^{2 m}, \quad p(2 m+1 \mid \rho)=0$,

where $r$ is a parameter and $m$ are all non-negative integers. In what follows we will consider just outcomes with an even number of photons. We can show that there are mixed classical thermal states $\Phi_{t}$ providing larger probability $P(\boldsymbol{n} \mid \Phi)$ than any pure coherent state $\Phi_{c}$. For thermal and coherent light with mean photon number $\bar{n}$ we have

$$
\begin{gathered}
p\left(2 m \mid \Phi_{t}\right)=\frac{1}{\bar{n}+1}\left(\frac{\bar{n}}{\bar{n}+1}\right)^{2 m}, \\
p\left(2 m \mid \Phi_{c}\right)=\exp (-\bar{n}) \frac{\bar{n}^{2 m}}{(2 m) !} .
\end{gathered}
$$

In Fig. 1 we have represented just the factors on $P\left(\boldsymbol{n} \mid \Phi_{t, c}\right)$ depending on $\Phi$

$$
P(\boldsymbol{n} \mid \Phi) \propto \exp \left[N \sum_{m=0}^{\infty} p(2 m \mid \rho) \ln p(2 m \mid \Phi)\right]
$$

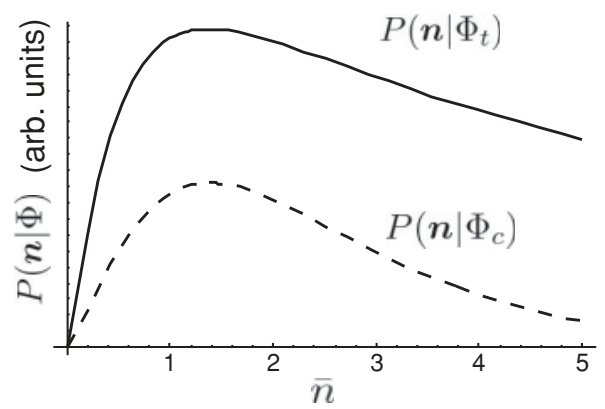

FIG. 1. Plot of $P\left(\boldsymbol{n} \mid \Phi_{t, c}\right)$ for a mixed thermal state (solid line) and for pure coherent states (dashed line) as functions of the mean number of photons $\bar{n}$ for the statistics of photon-number measurement in squeezed vacuum with $r=1$. 
for $N=1$ and $r=1$ as functions of $\bar{n}$. This shows that for mixed thermal states, $P(\boldsymbol{n} \mid \Phi)$ is larger than for pure coherent states. Therefore $\Phi_{n}$ cannot be pure.

2. Multiple-outcome classical bounds are below singleoutcome bounds: The maximization of the global probability $P(\boldsymbol{n} \mid \Phi)$ in terms of a single $\Phi_{n}$ is different from the independent maximization of the individual $p(m \mid \Phi)$, which leads to different $\Phi_{m}$ for different outcomes $m$. Therefore we have

$$
P\left(\boldsymbol{n} \mid \Phi_{n}\right) \leqslant N ! \Pi_{m=1}^{M} \frac{\left[p\left(m \mid \Phi_{m}\right)\right]^{n_{m}}}{n_{m} !},
$$

since by definition of $\Phi_{m}$ it holds $p\left(m \mid \Phi_{n}\right) \leqslant p\left(m \mid \Phi_{m}\right)$. This means that the multiple-outcome tests can be more effective in detecting nonclassicality than the single-outcome criteria.

Let us demonstrate this feature with a simple example of an angular momentum or spin $j=1$. For spins the classical states are the $\mathrm{SU}(2)$ coherent states $|j, \Omega\rangle$ and their incoherent superpositions with positive weights $[9,10]$

$$
\Phi=\int d \Omega P(\Omega)|j, \Omega\rangle\langle j, \Omega|, \quad P(\Omega) \geqslant 0,
$$

where

$$
\begin{aligned}
|j, \Omega\rangle= & \sum_{m=-j}^{j}\left(\begin{array}{c}
2 j \\
j+m
\end{array}\right)^{1 / 2}\left(\sin \frac{\theta}{2}\right)^{j-m} \\
& \times\left(\cos \frac{\theta}{2}\right)^{j+m} e^{-i m \phi}|j, m\rangle,
\end{aligned}
$$

$|j, m\rangle$ are the simultaneous eigenstates of $j^{2}$ and $j_{3}, \Omega=(\theta, \phi)$ are state parameters, and $d \Omega=\sin \theta d \theta d \phi$. The probabilities of the outcomes $m= \pm 1,0$ of $j_{3}$ for any $\rho$ can be expressed as

$$
p( \pm 1 \mid \rho)=\frac{1}{2}\left(\left\langle j_{3}^{2}\right\rangle \pm\left\langle j_{3}\right\rangle\right), \quad p(0 \mid \rho)=1-\left\langle j_{3}^{2}\right\rangle .
$$

For $j=1$ classical behavior [i.e., nonsingular positive $P(\Omega)$ ] is equivalent to a non-negative covariancelike matrix [10]

$$
Z_{k, \ell}=\left\langle\left(j_{k} j_{\ell}+j_{\ell} j_{k}\right)\right\rangle-\delta_{k, \ell}-\left\langle j_{k}\right\rangle\left\langle j_{\ell}\right\rangle .
$$

In particular, from the necessary condition $Z_{3,3} \geqslant 0$ we get that for classical states

$$
\left\langle j_{3}^{2}\right\rangle \geqslant \frac{1}{2}\left(1+\left\langle j_{3}\right\rangle^{2}\right) .
$$

For simplicity let us further assume $p(-1 \mid \rho)=0$, so that $p(1 \mid \rho)+p(0 \mid \rho)=1$. This is the case, for example, of states of the form, in the $|j, m\rangle$ basis,

$$
|\psi\rangle=\sqrt{p(1 \mid \rho)}|1,1\rangle+\sqrt{p(0 \mid \rho)}|1,0\rangle .
$$

The task is to maximize

$$
P(\boldsymbol{n} \mid \Phi) \propto[p(1 \mid \Phi)]^{n_{1}}[p(0 \mid \Phi)]^{n_{0}} .
$$

From Eqs. (23) and (25) for fixed $\left\langle j_{3}^{2}\right\rangle$ the largest $P(\boldsymbol{n} \mid \Phi)$ is obtained for the largest $\left\langle j_{3}\right\rangle$ compatible with classicality. From Eq. (25) this is

$$
\left\langle j_{3}^{2}\right\rangle=\frac{1}{2}\left(1+\left\langle j_{3}\right\rangle^{2}\right)
$$

which holds for SU(2) coherent states [11]. Thus, the relevant probabilities for classical states $\Phi$ can be expressed as

$$
p(1 \mid \Phi)=\frac{1}{4}\left(1+\left\langle j_{3}\right\rangle\right)^{2}, \quad p(0 \mid \Phi)=\frac{1}{2}\left(1-\left\langle j_{3}\right\rangle^{2}\right) .
$$

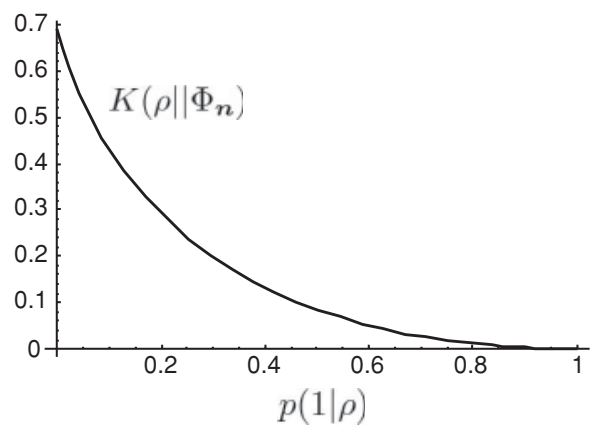

FIG. 2. Kullback-Leibler divergence $K\left(\rho \| \Phi_{n}\right)$ comparing the actual observed statistics $P(\boldsymbol{n} \mid \rho)$ and the closest statistics compatible with classical physics $P\left(\boldsymbol{n} \mid \Phi_{n}\right)$ given by Eqs. (29) and (30), as a function of $p(1 \mid \rho)$ showing nonclassicality $K\left(\rho \| \Phi_{n}\right)>0$ for all $p(1 \mid \rho) \neq 1$.

Note that $p(1 \mid \Phi)+p(0 \mid \Phi)<1$ because for classical states $\Phi$ we cannot force $p(-1 \mid \Phi)=0$ in general. After Eq (29) the maximum of $P(\boldsymbol{n} \mid \Phi)$ in Eq. (27) when $\left\langle j_{3}\right\rangle$ is varied holds for

$$
\left\langle j_{3}\right\rangle=\frac{n_{1}}{n_{0}+n_{1}}=p(1 \mid \rho) .
$$

Equations (29) and (30) determine completely the statistics compatible with classical physics $P\left(\boldsymbol{n} \mid \Phi_{n}\right)$ closest to the actual observed statistics $P(\boldsymbol{n} \mid \rho)$. We can compare them by computing the Kullback-Leibler divergence $K\left(\rho \| \Phi_{n}\right)$ which is represented in Fig. 2 as a function of $p(1 \mid \rho)$. This shows nonclassicality for all $p(1 \mid \rho) \neq 1$. Maximum nonclassicality holds for $p(1 \mid \rho)=0$, which arises when $\rho$ is the state $|j=1, m=0\rangle$, while the only case of classicality $p(1 \mid \rho)=1$ arises when $\rho$ is the $\mathrm{SU}(2)$ coherent state $|j=1, m=1\rangle$.

On the other hand, the classical upper bounds for the singleoutcome probabilities are [2]

$$
p\left(1 \mid \Phi_{1}\right)=1, \quad p\left(0 \mid \Phi_{0}\right)=\frac{1}{2}
$$

which hold when $\Phi_{1,0}$ are the polar and equatorial SU(2) coherent states with $\theta_{1,0}=0, \pi / 2$, respectively. Therefore, for all states with $p(-1 \mid \rho)=0$ and $1>p(1 \mid \rho) \geqslant 1 / 2$ we have $p(0 \mid \rho) \leqslant 1 / 2$, so that these statistics would be compatible with the single-outcome classical bounds (31), but incompatible with the multiple-outcome classical bound, as shown in Fig. 2.

Conclusions. We have derived simple practical tests to reveal the quantum nature of states by considering classical bounds on the statistics of multiple outcomes of an arbitrary measured observable. These nonclassical tests can be expressed in terms of the Kullback-Leibler divergence. Nonclassicality criteria for multiple outcomes can be fulfilled by states that do not satisfy the corresponding single-outcome nonclassical tests.

A.L. acknowledges Prof. Ph. Réfrégier and Dr. A. Rivas for their enlightening discussions. This work has been supported by Project No. FIS2008-01267 of the Spanish Dirección General de Investigación del Ministerio de Ciencia e Innovación, and by Project QUITEMAD S2009-ESP-1594 of the Consejería de Educación de la Comunidad de Madrid. 
[1] L. Mandel and E. Wolf, Optical Coherence and Quantum Optics (Cambridge University, Cambridge, England, 1995); M. O. Scully and M. S. Zubairy, Quantum Optics (Cambridge University, Cambridge, England, 1997); Ch. Gerry and P. Knight, Introductory Quantum Optics (Cambridge University, Cambridge, England, 2004); V. V. Dodonov, J. Opt. B 4, R1 (2002).

[2] A. Rivas and A. Luis, Phys. Rev. A 79, 042105 (2009).

[3] E. C. G. Sudarshan, Phys. Rev. Lett. 10, 277 (1963); R. J. Glauber, Phys. Rev. 131, 2766 (1963).

[4] J. G. Kirkwood, Phys. Rev. 44, 31 (1933); H. Margenau and R. N. Hill, Prog. Theor. Phys. 26, 722 (1961); C. L. Mehta, J. Math. Phys. 5, 677 (1964); L. Cohen, ibid. 7, 781 (1966); K. E. Cahill and R. J. Glauber, Phys. Rev. 177, 1857 (1969); 177, 1882 (1969); G. S. Agarwal and E. Wolf, Phys. Rev. D 2, 2161 (1970); 2, 2187 (1970); 2, 2206 (1970).

[5] J. A. Vaccaro, Opt. Commun. 113, 421 (1995); Phys. Rev. A 52, 3474 (1995); J. A. Vaccaro and D. T. Pegg, ibid. 41, 5156 (1990); L. M. Johansen, Phys. Lett. A 329, 184 (2004); J. Opt. B 6, L21 (2004); L. M. Johansen and A. Luis,
Phys. Rev. A 70, 052115 (2004); A. Luis, ibid. 73, 063806 (2006).

[6] T. M. Cover and J. A. Thomas, Elements of Information Theory (Wiley Interscience, New York, 1991).

[7] P. Marian, T. A. Marian, and H. Scutaru, Phys. Rev. A 69, 022104 (2004).

[8] L. Mandel, Phys. Scr., T 12, 34 (1986); M. Hillery, Phys. Rev. A 35, 725 (1987); C. T. Lee, ibid. 44, R2775 (1991); 52, 3374 (1995); M. G. Benedict and A. Czirják, ibid. 60, 4034 (1999); V. V. Dodonov, O. V. Man'ko, V. I. Man'ko, and A. Wünsche, J. Mod. Opt. 47, 633 (2000); W. Vogel, Phys. Rev. Lett. 84, 1849 (2000); P. Marian, T. A. Marian, and H. Scutaru, ibid. 88, 153601 (2002); J. M. C. Malbouisson and B. Baseia, Phys. Scr. 67, 93 (2003); V. V. Dodonov and M. B. Renó, Phys. Lett. A 308, 249 (2003); J. K. Asbóth, J. Calsamiglia, and H. Ritsch, Phys. Rev. Lett. 94, 173602 (2005).

[9] F. T. Arecchi, E. Courtens, R. Gilmore, and H. Thomas, Phys. Rev. A 6, 2211 (1972).

[10] O. Giraud, P. Braun, and D. Braun, Phys. Rev. A 78, 042112 (2008).

[11] A. Luis and N. Korolkova, Phys. Rev. A 74, 043817 (2006). 